\title{
Methodologies for Scale-down of Microbial Bioprocesses
}

\section{Maria Papagianni*}

Department of Hygiene and Technology of Food of Animal Origin, School of Veterinary Medicine, Aristotle University of Thessaloniki. Thessaloniki 54006, Greece

\begin{abstract}
Much research has been done on bioreactor hydrodynamics and their interactions with various microbial cell mechanisms and several methodologies have been proposed in order to resolve scale-up problems. Among them is the approach of scale-down and regime analysis. Regime analysis at production scale has to define the rate-limiting mechanisms of the process and identify the ruling regime. Such an analysis, based on characteristic times, can give valuable information for scale translation and optimization of bioprocesses. An efficient scale-down bioreactor has to create conditions that will be representative for the conditions occurring at large scale. Miniaturised bioreactor (MBR) systems may serve as scale-down tools especially in early-stage process operations. In later stages, e.g. optimization of process conditions and operations, only scaleable apparatus can be applied successfully. The choice of the scale-down method, and therefore the kind of the applied scale-down bioreactor, should be based on the characteristics of the process. The constructed models and the rules used to scale-down the process will be used in scaling-up the optimized conditions at production scale. The paper reviews the systematic approach of scale-down methodologies.
\end{abstract}

\section{Introduction}

Laboratory-scale bioreactors typically have a working volume that varies from about $0.2 \mathrm{~L}$ to $20 \mathrm{~L}$. Such bioreactors can be used with a high power input, resulting in rapid mixing of the fermentation broth and high mass-transfer rates. Only systems of increased viscosity, like filamentous mycelial broths, or shear-sensitive systems, like plant cells, will give mass, heat and momentum transfer problems on such a small scale. On a production-scale, the power input is restricted for economical and mechanical reasons, causing mass and heat-transfer problems. Large-scale industrial bioprocesses with cultivation volumes ranging from 10,000 to $500,000 \mathrm{~L}$ are generally inhomogeneous and concentration profiles of nutrients, cells and products are characterized by large gradients. Cells in large-scale bioreactors are forced to travel through different zones inside the reactor and to experience a variety of conditions, e.g. oxygen limitations, increased substrate concentrations, $\mathrm{pH}$ shifts, shear forces, in which they respond [1-3]. Under such conditions, their physiology will differ from that of cells growing in homogeneous, well-mixed cultures. Obviously, scale-up of bioprocesses introduces these problems.

In their important review on regime analysis and scale-down, Sweere and co-workers [4] suggested two ways to solve the problems arising during scale-up:

a. By acquiring more knowledge on the hydrodynamics of largescale bioreactors and their interactions with other mechanisms, in order to get a complete description of the system;

b. By developing scale-up procedures that give an adequate estimation of the performance of production-scale bioreactors based on small-scale investigations.

Much research has been done on bioreactor hydrodynamics and various models have been proposed in order to resolve scale-up problems. Various scale translation approaches are based on constant impeller tip speed $\left(\mathrm{ND}_{\mathrm{i}}\right)$, constant power per unit volume $(\mathrm{Pg} / \mathrm{V})$ and constant mixing time $\left(\mathrm{t}_{\mathrm{m}}\right)$ [5]. For years scale-up was based on experience, trial and error, and rules of thumb. Later it developed to a more systematic procedure and a number of methods have been discussed in the literature. These include the following:

1. Fundamental method;
2. Semi-fundamental method;

3. Dimensional analysis / regime analysis;

4. Rules of thumb;

5. Scale-down / regime analysis;

6. Trial and error;

7. Multiplication of elements.

Generally, a large number of rules of thumb and scale-up parameters have been described and have been used with varying degrees of success. Often combinations of different methods can yield good results [4]. The semi-fundamental method in combination with rules of thumb has been the most widespread method. Scale-down of rate-limiting mechanisms based on the results of regime analysis is a powerful tool to solve scale-up problems. However, only a few methodologies have been suggested for scaling-down processes to the laboratory scale to investigate the performance of bioreactors and the parameters that significantly affect the culture. These methodologies, and the new approaches, will be reviewed in this work.

\section{The scale-down approach}

Oosterhuis [6] described the four-step scale-down procedure (Figure 1) as follows:

1. Regime analysis of the process at production scale;

*Corresponding author: Maria Papagianni, Department of Hygiene and Technology of Food of Animal Origin, School of Veterinary Medicine, Aristotle University of Thessaloniki. Thessaloniki 54006, Greece, E-mail: mp2000@vet. auth.gr

Received July 11, 2011; Accepted October 29, 2011; Published November 04 2011

Citation: Papagianni M (2011) Methodologies for Scale-down of Microbial Bioprocesses. J Microbial Biochem Technol S5:001. doi:10.4172/1948-5948.S5001

Copyright: ( 2011 Papagianni M. This is an open-access article distributed under the terms of the Creative Commons Attribution License, which permits unrestricted use, distribution, and reproduction in any medium, provided the original author and source are credited 
2. Simulation of the rate-limiting mechanisms at laboratory scale;

3. Optimization and modelling of the process at laboratory scale;

4. Implementation of the process at production scale by transformation of the optimized laboratory conditions.

The first step in the scale-down approach involves a regime analysis of the process at production scale. A consistent methodology is needed in order to analyse all the sub-processes involved in the system. Detailed description of the sub-processes results to large sets of mathematical expressions that describe the time-dependence of a large number of variables [7]. The complex reality should then be incorporated into a model that is able to describe accurately the various mechanisms involved. Regime analysis has to define the rate-limiting mechanisms of the process and identify the ruling regime which could be pure regime (regime ruled by one mechanism) or mixed regime (more mechanisms) [4].

The most important requirement for experiments at laboratory scale is the fact that they have to be representative for the conditions prevailing at the large scale. This determines the limits of laboratoryscale experiments. The next step of optimization has again its limitations since not all possible optimization results can be used because the optimized situation will be translated back to the production scale. At this step, data obtained from growth and product formation analyses as well as of hydrodynamic analysis will be used to construct models. These models should ideally be capable to contribute to the understanding of microbial dynamics and predict growth and product formation under the transient conditions of large-scale situations. In the last step, the optimized laboratory conditions are implemented at production scale. Models constructed in the previous step can be applied and rules used to scale-down the process conditions can be used in scaling-up these conditions again.

Regime analysis: The "regime" concept was introduced by Johnstone and Thring [8] as a necessary ingredient for the solution of engineering problems by means of dimensional analysis based on the similarity principle. Although the concept was not initially demonstrated for scale-up purposes, it has been extensively treated in the relative literature and is one of the fundamental methods of scaleup.

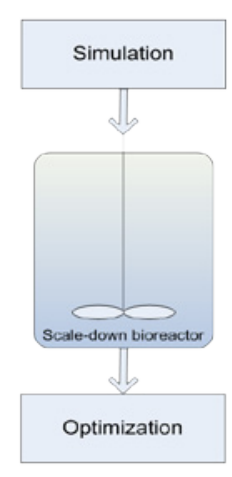

Experimental scal

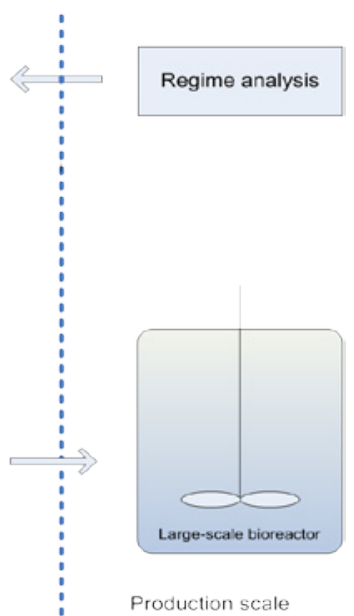

Production scale
Figure 1: The four-step scale-down procedure.
Regime analysis must provide information about the performance of the process. This can be achieved through a four-step procedure $[9,10]$ that involves:

1. Decision-definition of the significant process variables;

2. Quantification of the process variables by adequate measurements;

3. Derivation of rate constants, e.g. Characteristic values of rates or time constants;

4. Comparisons of these values with the result that the slowest rate (largest time) governs the overall process rate (= rate determining step).

Therefore, the information about the process performance through regime analysis is based on a comparison of the rate of the mechanisms which may play a role in the process. The comparison can be made experimentally or theoretically and quantitatively or qualitatively.

Examples of experimental regime analysis can be found in the works of Kossen and Oosterhuis [11], Moes et al. [12], Levespiel [13] and Amanullah et al. [14]. However, not all the problems that have to be solved can be met only by experimental regime analysis. The question whether change of regime will occur during scale-up can only be answered through theoretical regime analysis.

Theoretical regime analysis includes regime analysis based on characteristic parameters (e.g. characteristic times) and parameter sensitivity analysis. The first is based on a comparison of the rates of different mechanisms, expressed in characteristic parameters that can often be obtained accurately by the use of rules of thumb. A comparison of these characteristic parameters will yield the rate-limiting mechanisms, or regime of the process. Parameter sensitivity analysis can be performed using a number of tools and methods, e.g. global or local sensitivity analysis. Sensitivity analysis techniques quantify how the output from a model depends upon each of its input variables. Global sensitivity, in particular, attempts to determine the relative effect of variables on the model outputs (all variables are analyzed simultaneously) in contrast with local sensitivity analysis, which typically determines the rate of change of model output with respect to individual model variables. Global sensitivity analysis therefore determines sensitivities of a multidimensional system whereas local sensitivity analysis determines a gradient at the operating point with respect to a single variable. In addition, global sensitivity techniques may be used to determine the strength of interactions between variables. The selection of appropriate operating conditions for a given bioprocess is complicated by the large number of interacting processing stages and variables. Once key variables have been determined, the most significant subset can be identified. Examples of application of parameter sensitivity analysis can be found in the works of Clarkson et al. [15], King et al. [16] and Chhatre et al. [17]. Here, only regime analysis based on characteristic times will be further discussed.

Characteristic times: In regime analysis based on "characteristic times" these are used to characterize the sub-processes of a system. Characteristic time is a measure of the rate of a mechanism and it can be considered as the time needed by that mechanism to smooth out a change to a certain fraction [4]. A low value of a characteristic time refers to a fast mechanism, while conversely, a high value to a slow mechanism. 
Characteristic times are also called "relaxation times" and "time constants". However, the term time constant is rather used for first order linear processes and it can be composed from the time constants of several mechanisms. If the process is not a first order linear one, the time constant cannot strictly defined in this way. Instead, characteristic times, introduced to characterize the rate of mechanisms, can be used to characterize non-linear and processes of a higher order with a single characteristic time, e.g. characterization of mixing in bioreactors by means of the mixing time. Characteristic times of a process can be determined by a number of methods, experimentally or theoretically. Analysis of the mathematical solution of a process model is a method which can be fairly simple for liner, first order differential equations. However, in the case of problems with no available solution to the differential equations or in the case of a problem for which a general differential equation cannot be defined, the procedure becomes complicated. The characteristic times of differential equations are generally regarded as more accurate but require solution of the equations and have the drawback that they are based on the estimation of various parameters. Parameters like mass transfer coefficients or dispersion, diffusion coefficients and others, can be estimated by correlations from the literature or from small-scale experiments. In the case the parameters are not known from the literature, reaction kinetics have to be estimated from experimental work.

Examples of experimental determination of the characteristic times mixing time $\left(\mathrm{t}_{\mathrm{m}}\right)$ and circulation time $\left(\mathrm{t}_{\mathrm{c}}\right)$ in mechanically agitated bioreactors can be found in the works of Einsele [18], Oosterhuis [6], Papagianni [19] and Papagianni et al. [20,21].

Theoretical determination of characteristic times of physical mechanisms in bioreactors can be done by correlations from the literature and rules of thumb. In the case of mixing for example:

$$
\mathrm{t}_{\mathrm{m}}=4 \mathrm{t}_{\mathrm{c}}
$$

$$
\text { and }
$$

$$
\mathrm{t}_{\mathrm{c}}=\frac{V}{2 H N \pi^{2} D^{2}}
$$

Apart from the characteristic times referring to the physical processes in a bioreactor, characteristic times that refer to the microorganisms and the physiological mechanisms of the system have to be estimated. A characteristic time can be chosen for any metabolic variable of the system. However, because of the dynamic nature of the conditions and the growing complexity with time, the description of the system should be limited to a few sub-processes.

For a batch system and balanced growth, Michaelis-Menten kinetics can be used for the estimation of the characteristic times of substrate consumption and product formation:

$$
\frac{d C_{s}}{d t}=\frac{\mu_{\max }}{Y_{s x}} \frac{C_{s} C_{x}}{K_{s}+C_{s}}
$$

or

$$
t_{s c}=\frac{C_{s}}{r_{s}}=\frac{Y_{s x}}{\mu_{\max } C_{x}}\left(K_{s}+C_{s}\right)
$$

Under dynamic conditions kinetics deviate from the above equations since mechanisms inside the cells may change the performance of the cultures resulting in non-balanced growth. In this case, characteristic times of microorganisms should be compared with characteristic times of environmental changes. Microorganisms have large capacities to react to environmental changes and therefore a wide range of characteristic times can be obtained in a way that is analogous to the order of magnitude of the environmental changes to which they are exposed [4].

There are several examples of the use of characteristic times in the literature. Among the most successful and extensively treated, is the example of regime analysis based on characteristic times for gluconic acid production by Gluconobacter oxydans in aerobic batch process as presented by Oosterhuis [6] and Kossen and Oosterhuis [11]. The process was carried out at the scale of about $25 \mathrm{~m}^{3}$. The rate limiting steps of the process were identified by regime analysis. Substrate consumption $\left(5.5 \times 10^{4} \mathrm{~s}\right)$ and growth $\left(1.2 \times 10^{4} \mathrm{~s}\right)$ were found to have no influence on process performance, while the characteristic times for oxygen consumption and oxygen transfer to the liquid phase were of the same order of magnitude (zero order). As the broth circulation time was also of the same order of magnitude, it was concluded that oxygen limitation and gradients were likely to occur. The oxygen gradients and local oxygen limitation were confirmed by measurements during fermentation in the $25 \mathrm{~m}^{3}$ reactor. Prediction of circulation time was checked also by measurements during flowfollower experiments. Other estimated characteristic times included: gas residence time, transfer of oxygen from a gas bubble, heat transfer and heat production. The regime analysis carried out for the particular system formed the basis of small-scale investigations on the influence of fluctuating oxygen concentrations on acid production and also in mass transfer studies. Scale-down was based on characteristic times for broth circulation and oxygen consumption.

Another example of regime analysis based on characteristic times is the one presented by Sweere [22] for the baker's yeast process in a bubble column bioreactor of $150 \mathrm{~m}^{3}$ with a working volume of 120 $\mathrm{m}^{3}$, in a study of the influence of transient environmental conditions on growth and metabolite production. Characteristic times in that case were calculated as a function of the gas flow rate. The particular process is sensitive to changes in oxygen and glucose concentrations (Pasteur and Grabtree effects, respectively) and it is important to know whether limitations or gradients of oxygen and glucose will occur. A distinction was made between mechanisms that have a clear effect on the process and those with a much smaller or larger characteristic time. The first included: mixing, liquid circulation, gas flow, oxygen transfer, substrate consumption, oxygen consumption and substrate addition. Parameters which were not relevant to the final performance of the bioreactor were the following: fed-batch process, growth of biomass, heat transfer, heat production and micro-mixing. This approach yielded valuable information about changing regimes during process optimization, scale-up or as the process proceeds.

Regime analysis based on characteristic times is a valuable tool in process optimization as it identifies and gives information about the rate-limiting mechanisms and the limitations and concentration gradients. From the retrieved information, it can be decided what further knowledge should be acquired and what experiments are needed to optimize the process.

\section{Experimental simulation of large-scale conditions: The scale- down bioreactor}

Data obtained from hydrodynamic analyses and modelling of largescale bioreactors has been used in many cases to elaborate scale-down 
bioreactors that have the capacity to reproduce the hydrodynamics encountered at the industrial scale. Scale-down reactors of various configurations have been described in the literature. Examples include the following: a stirred tank bioreactor with separated internal compartments [23-25], a stirred tank bioreactor with randomly fluctuating substrate addition [26,27], a stirred tank bioreactor connected to a plug-flow section [2,14,28], two or more bioreactors in series [29-31], and tubular loop bioreactors [19,23].

From the regime analysis of the gluconic acid production process (see previous section), Oosterhuis [6] concluded that the culture is exposed to a continually changing oxygen concentration and scaledown was based on characteristic times for broth circulation and oxygen consumption. Two segment models were used for experimental simulation: the first consisted of a batch bioreactor with periodically changing inlet gas concentration (from air to nitrogen gas); the second consisted of two reactors one sparged with air, the other with nitrogen, with an exchange flow between them. In the first model, a reduction in productivity occurred due to anaerobic periods and a negative effect on the potential capacity of the cells to produce gluconic acid was observed. In the second model of two bioreactors and circulation from the aerobic to anaerobic environment, no effect was observed on the potential capacity of the cells to produce gluconic acid. The reduction of the overall process productivity was attributed to the anaerobic volume fraction of the process.

More recently, a methodology for the design of scale-down bioreactors was presented by Delvigne and co-workers [32] who applied mixing and circulation stochastic models that permitted an insight on the internal dynamics of the process. Stochastic analysis of large-scale bioreactors permitted to propose a translating methodology into scale-down context. Stirred vessels of three different volumes were used for mixing time experiments: a $30 \mathrm{~L}$ vessel, a $500 \mathrm{~L}$ bioreactor and a $2000 \mathrm{~L}$ bioreactor. The standard scale-down device was a $30 \mathrm{~L}$ vessel, assumed to be perfectly mixed, which was connected to a plug-flow section (Figure 2). Circulation of the liquid between the mixed and the plug-flow sections was ensured by the use of a peristaltic pump. Four different kinds of scale-down apparatus which differed in the design of the plug-flow section were studied: a silicone tubing of $8 \mathrm{~mm}$ internal diameter and $7.5 \mathrm{~mm}$ length, a glass bulb of $85 \mathrm{~mm}$ internal diameter and $0.25 \mathrm{~m}$ length, two glass bulbs in series and two glass bulbs in

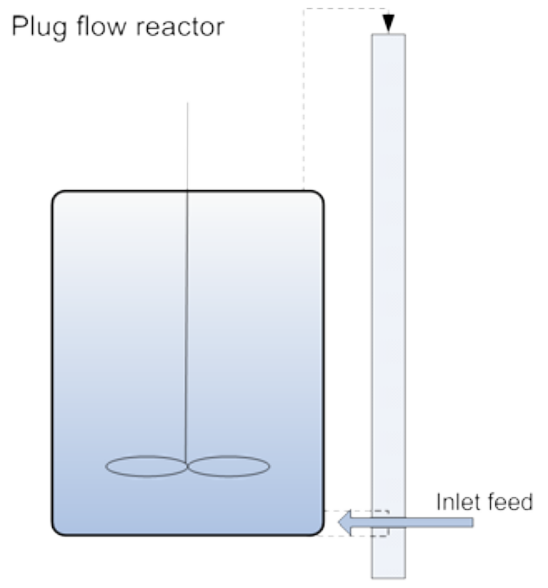

Figure 2: Plug-flow reactor. parallel. By using stochastic models, the authors proposed a simple procedure to translate the hydrodynamic characteristics of a large-scale bioreactor at the level of a scale-down reactor comprising a perfectly mixed and a plug-flow part. The procedure involved two steps: the first step led to the determination of a concentration field at a given homogeneity degree, while the second involved the use of structured parameters derived from stochastic simulations. Both steps led to the calculus of structured criteria which represented efficiently the mixing and circulation capacities of the system. By superimposing the two structured criteria the authors obtained the profile of concentrations into which a microorganism was subjected during its cultivation in the bioreactor.

The profile of carbon source concentrations was the subject for simulations in the work of Dodge and co-workers [33]. The authors described a method to simulate substrate gradients in production-scale fed-batch bioreactors by pulse feeding of the substrate in laboratory bioreactors. Three different methods of reducing mixing time in the manufacturing (large-scale) equipment were applied: a) increasing the agitation rate, b) replacing radial flow with axial flow impellers, and c) adding feed at additional points within the reactor. Implementation of the suggested solutions at large scale resulted in process improvements and a performance which was closer to that of laboratory-scale.

Dissolved carbon dioxide gradients $\left(\mathrm{dCO}_{2}\right)$, which van occur in large-scale reactors, were simulated for the first time by Baez and co-workers [34] and their effects were evaluated on Escherichia coli expressing recombinant green fluorescent protein. $\mathrm{dCO}_{2}$ gradients were simulated by continuously circulating the medium between two vessels of a scale-down system to mimic mean circulation times of 50, 170 , and $375 \mathrm{~s}$. The authors showed that while exposure to elevated $\mathrm{dCO}_{2}$ can affect culture performance, E. coli can rescue its metabolism in short times when cells are intermittently returned to low $\mathrm{dCO}_{2}$ conditions after being exposed to high $\mathrm{dCO}_{2}$

In order to analyze and optimize the performance of a microbial process, an integrated model of bioreactor hydrodynamics and microbial kinetics is needed. Having constructed such a model, performance could be calculated with bioreactors of different geometries and cultivation technologies. The basis of the model however, has to be measurements of the actual process. These measurements have to give the dynamic response of the cells to the micro-environment of the chosen bioreactor. Very often, the behaviour of the microbial culture becomes the bottleneck of the process and the cause of engineering problems, especially when filamentous microorganisms with transient morphologies, and subsequently physiological states, are employed. Under such conditions, it is difficult for an analysis of engineering problems to be based on dimensionless numbers only, or on any other method, neglecting the morphology of the producer organism. In an effort to study the response of a filamentous system to the changing conditions inside large stirred tank bioreactors, Papagianni [19] and Papagianni et al. [20,35] used a tubular loop bioreactor which was tested using batch cultivations of the citric acid producing filamentous fungus Aspergillus niger, as a test system.

The loop reactor is a well known reactor type in the chemical process technology. Its characteristics, e.g. simple design, defined flow paths, large mass transfer rates for substrates and oxygen at low energy inputs, attracted research for its suitability as bioreactor [36-38]. Moreover, reports have shown that loop configurations simulated successfully stirred tanks [39-41]. In these works however, comparisons were based on growth kinetics and production levels. 
When filamentous microorganisms were used, morphology was assessed only qualitatively or was not given any consideration at all. In this direction, the work of Papagianni et al. [21] reported the design and construction of a loop bioreactor (Figure 3) for studies on scaleup of non-Newtonian mycelial suspensions. Broth circulation around the loop was done by peristaltic pumps and the circulation time $\left(t_{c}, s\right)$ was used as a measure of simulated reactor size. The reactor carried a number of ports and probes along its length for the determination of concentration gradients within. The system was evaluated using the citric acid fermentation by $A$. niger as test process, while investigations were focused on relationships between circulation time, morphological parameters, and citric acid production, using the latter as a measure of reactor performance. Table 1 shows the characteristic times for circulation and mixing times of the particular loop reactor and the citric acid fermentation process by $A$. niger. From comparative experiments carried out in 10 and $200 \mathrm{~L}$ stirred tank bioreactors, it appeared that the loop reactor simulated the corresponding stirred tanks (Figure 4)

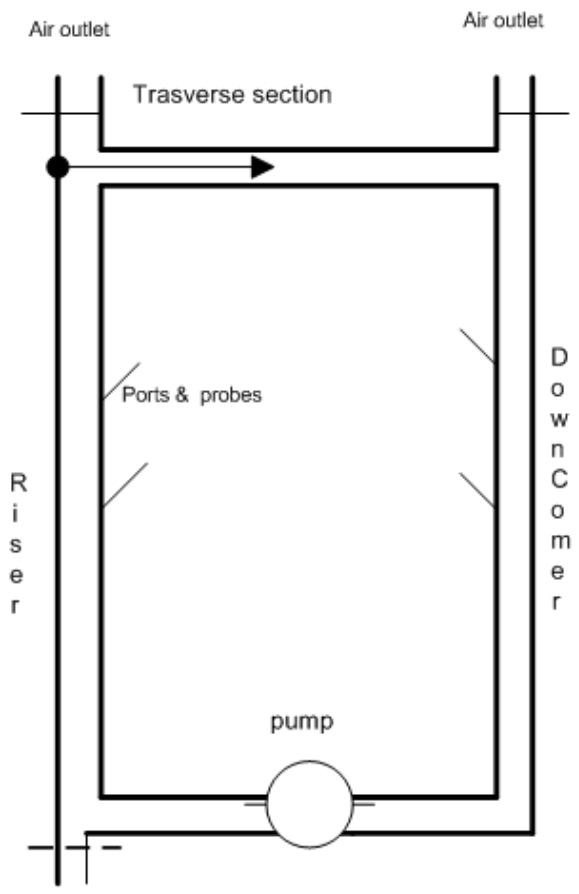

Figure 3: The tubular loop bioreactor (Papagianni et al. 2003).

\begin{tabular}{|c|c|c|c|}
\hline Apparent viscosity (cP) & Pump setting & Circulation time (s) & Mixing time (s) \\
\hline 1 & 99 & 15 & 72 \\
\hline 10 & 99 & 15 & 77 \\
\hline 15 & 99 & 15 & 61 \\
\hline 22 & 99 & 15 & 67 \\
\hline 40 & 99 & 15 & 69 \\
\hline 40 & 75 & 17 & 72 \\
\hline 40 & 50 & 30 & 100 \\
\hline 40 & 25 & 59 & 120 \\
\hline
\end{tabular}

Table 1: : Characteristic times $(t, t, s)$ of the loop bioreactor presented in the work of Papagianni et al. [21] in the citric acid fermentation process by $A$. niger.

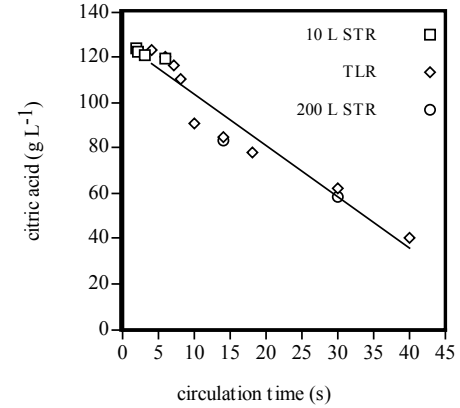

Figure 4: The influence of circulation time $(t, s)$ on citric acid production in a 10 L stirred tank. bioreactor (STR), in a 200 L STR and in the loop bioreactor used by Papagianni et al. [21]

representing a valuable tool in scaling-up and -down of the particular fermentation process.

\section{Current trends: Miniature bioreactors as a scale-down tool}

The progress in molecular biology and genetic engineering and the need to carry out a large number of development cultivations especially in the pharmaceutical / healthcare research and development, has resulted in the advance of ultra small volume bioreactors, the miniature bioreactors (MBRs), with working volumes in the range of tens of $\mu \mathrm{l}$ to $100 \mathrm{ml}$, that appear to have increasingly widespread applications. The subject has been extensively discussed by Betts and Baganz [42] and Neubauer and Junne [43].

MBRs are currently based on existing bioreactor configurations e.g. shake flasks, stirred tank and bubble column reactors. They may have a high degree of automation although, they are currently less instrumented than conventional laboratory-scale bioreactors. Also, the small working volumes limit sampling opportunities. MBRs aim to accelerate research, reduce costs and labour intensity and apart from the therapeutic drugs development, there have been several other applications e.g. in growth media development, strain improvement, metabolic engineering studies and others. From the view of process development, these applications are early-stage operations. In later stages, e.g. optimization of process conditions and operations, only scaleable MBRs can be applied successfully. The choice of the scaledown method, and therefore the kind of the applied MBR, should be based on the characteristics of the process.

In bioprocesses carried out by fast-growing microorganisms like E. coli, oxygen transfer is usually the limiting factor and therefore the specific power input or the $k_{L} a$ can be used as scale-down criteria. Studies carried out by Betts et al. [44] on cultivations of a plasmid DNA producing $E$. coli strain in a miniature $10 \mathrm{ml}$ stirred tank bioreactor showed scale-down equivalence with a conventional $7 \mathrm{~L}$ stirred tank bioreactor. The scale-down criterion in that work was the specific power input. The results also revealed a high degree of equivalence in the $k_{L} a$ values obtained in both scales. Another example can be found in the work of Doig et al. [45]. Using a correlation for $k_{L} a$ predictions in microtitre plate systems (MTPs) they were able to scale-up the cultivation of $E$. coli that over-expressed a transketolase enzyme, from a $1 \mathrm{ml}$ MTP system to a $1.4 \mathrm{~L}$ stirred tank reactor using the criterion of constant $k_{L} a$. Other examples of successful applications of MBRs as scale-down tools using the $k_{L} a$ criterion can be found in the works of Gill et al. [46-48]. 
Citation: Papagianni M (2011) Methodologies for Scale-down of Microbial Bioprocesses. J Microbial Biochem Technol S5:001. doi:10.4172/19485948.S5-001

The field of miniature bioreactors is expanding rapidly. As automation of MBRs is the key to expanding capabilities, recently appeared devices are equipped with sophisticated in-built robotics and offer a great deal of promise for delivering fully integrated, highthroughput solutions. It is impossible however at the moment, by using MBRs only to solve the multidimensional problem of scale-up of bioprocesses. The agitation systems employed in MBRs (usually magnetic stirrers) result in flow regimes that are different from those found in traditional laboratory-scale bioreactors and this is translated into large differences in mixing and mass transfer and therefore to difficulties in reproducibility. Also, in processes in which further steps are required for product recovery, the use of MBRs along with miniaturized downstream processing devices as scale-down tools may be insufficient because of the very small volumes employed. Therefore, there is no single MBR that offers a solution to all problems.

\section{Conclusions}

- Scale-down of rate limiting mechanisms based on regime analysis of large-scale microbial bioprocesses represents an alternative method and a powerful tool to solve scale-up problems.

- From the knowledge gained from regime analysis it can be decided which mechanisms and features need to be investigated and optimized on a laboratory scale.

- Various experimental set-ups can be used to simulate industrial-scale fermentation processes on laboratory scale. A successful scale-down bioreactor must have the capacity to reproduce the hydrodynamics encountered at the industrial scale, as well as the behaviour of the culture, especially in the case of filamentous fermentations.

\section{List of symbols}

\begin{tabular}{|c|c|c|}
\hline $\mathrm{C}_{\mathrm{s}}$ & concentration of substrate & $\mathrm{kg} \mathrm{m}^{-3}$ \\
\hline $\mathrm{C}_{\mathrm{x}}$ & concentration of biomass & $\mathrm{kg} \mathrm{m}^{-3}$ \\
\hline $\mathrm{D}$ & diameter & $\mathrm{m}$ \\
\hline $\mathrm{D}_{\mathrm{i}}$ & Impeller diameter & $\mathrm{m}$ \\
\hline $\mathrm{H}$ & height & $\mathrm{m}$ \\
\hline $\mathrm{K}_{\mathrm{s}}$ & Michaelis-Menten constant & $\mathrm{kg} \mathrm{m}^{-3}$ \\
\hline $\mathrm{k}_{\mathrm{L}} \mathrm{a}$ & oxygen transfer coefficient & $\mathrm{h}^{-1}$ \\
\hline $\mathrm{N}$ & stirrer speed & $\mathrm{s}^{-1}$ \\
\hline $\mathrm{P}$ & power input & $\mathrm{W}$ \\
\hline $\mathrm{V}$ & volume & $\mathrm{m}^{3}$ \\
\hline$r_{s}$ & substrate consumption rate & $\mathrm{kg} \mathrm{m}^{-3} \mathrm{~s}^{-1}$ \\
\hline $\mathrm{t}$ & time & $\mathrm{s}$ \\
\hline$t_{c}$ & circulation time & s \\
\hline $\mathrm{t}_{\mathrm{m}}$ & mixing time & s \\
\hline $\mathrm{Y}_{\mathrm{sx}}$ & yield of biomass on substrate & kg dry wt kg-1 \\
\hline$\mu_{\max }$ & maximum specific growth rate & $\mathrm{h}^{-1}$ \\
\hline
\end{tabular}

\section{References}

1. Enfors SO, Jahic M, Rozkov A,Xu B, Hecker M, et al.(2001) Physiological responses to mixing in large scale bioreactors. J Biotechnol 85: 175-185.

2. Neubauer L, Häggstrom L, Enfors SO (1995) Influence of substrate oscillations on acetate formation and growth yield in Escherichia coli glucose limited fedbatch cultivations. Biotechnol Bioeng 47: 139-146.
3. Schmidt F (2005) Optimization and scale-up of industrial fermentation processes. Appl Microbiol Biotechnol 68: 425-435.

4. Sweere APJ, Luyben KCAM, Kossen NWF (1987) Regime analysis and scaledown: tools to investigate the performance of bioreactors. Enzyme Microb Technol 9: 386-398.

5. Vrabel P, van der Lans RGJM, Luyben KCAM, Boon L, Nienow AW (2000) Mixing in large-scale vessels stirred with multiple radial or radial and axial uppumping impellers: modelling and measurements. Chem Eng Sci 55: 5881 5896.

6. Oosterhuis NMG (1984) Scale-down of bioreactors. Ph.D. Thesis. Delft University of Technology. The Netherlands.

7. Roels JA (1983) Energetics and kinetics in biotechnology. Elsevier Biomedical. Amsterdam.

8. Johnstone RE, Thring MW (1957) Pilot plants, models and scale-up methods in chemical engineering, McGraw-Hill New York.

9. Moser A (1991) Strategies in bioreactor performance studies. Bioprocess Eng 6: 205-211.

10. Sweere APJ, Dalen JP, Kishoni E, Luyben KCAM, Kossen NWF (1989) Theoretical analysis of baker's yeast production: An experimental verification as a laboratory scale. Bioprocess Eng 3: 11-17.

11. Kossen NWF, Oosterhuis NMG, Rehm HJ, Reed G, VCH Verlagsgesellschaft (1985) Modelling and scale-up of bioreactors. In: (eds). Biotechnology. 2: 572 605.

12. Moes J, Griot M, Keller J, Heinzle E, Dunn IJ, et al. (1985) A microbial culture with oxygen-sensitive product distribution as a potential tool for characterizing bioreactor oxygen transport. Biotechnol Bioeng 27: 482-489.

13. Levenspiel O (1999) Chemical reaction engineering. $3^{\text {rd }}$ ed. John Wiley \& Sons. New York

14. Amanullah A, Mc Farlane CM, Emery AN, Nienow AW (2001) Scale-down model to simulate spatial $\mathrm{pH}$ variations in large-scale bioreactors. Biotechno Bioeng 73: 390-399.

15. Clarkson Al, Bulmer M, Titchener-Hooker NJ (1996) Pilot-scale verification of a computer-based simulation for the recovery of biological particles. Bioprocess Eng 14: 81-89

16. King JMP, Titchener-Hooker NJ, Zhou Y (2007) Ranking bioprocess variables using global sensitivity analysis: a case study in centrifugation. Bioprocess Biosystem Eng 30: 123-134.

17. Chhatre S, Francis R, Newcombe AR, Zhou Y, Titchener-Hooker N, et al. (2008) Global Sensitivity Analysis for the determination of parameter importance in bio-manufacturing processes. Biotechnol Appl Biochem 51: 79-90.

18. Einsele A (1976) Characterization of bioreactors by mixing times. Chem Rundschau 29: 53-55.

19. Papagianni M (1995) Morphology and citric acid production of Aspergillus niger in submerged fermentation. Ph.D. Thesis. University of Strathclyde. United Kingdom.

20. Papagianni M, Mattey M, Kristiansen B (1998) Citric acid production and morphology of Aspergillus niger as functions of the mixing intensity in a tubular loop and a stirred tank bioreactor. Biochem Eng J 2: 197-205.

21. Papagianni M, Mattey M, Kristiansen B (2003) Design of a tubular loop bioreactor for scale-up and scale-down of fermentation processes. Biotechnol Prog 19: 1498-1504

22. Sweere APJ (1988) Response of baker's yeast to transient environmenta conditions relevant to large-scale fermentation processes. Ph.D. Thesis. Delf University of Technology. The Netherlands.

23. Schilling BM, Pfefferle W, Bachmann B, Leuchtenberger W, Decker WD (1999) A special reactor dsign for investigations of mixing time effects in a scaleddown industrial L-lysine fed-batch fermentation process. Biotechnol Bioeng 64 599-606.

24. Glazyrina J, Materne E, Hillig F, Neubauer P, Junne S (2011) Two-compartmen method for determination of the oxygen transfer rate with electrochemical sensors based on sulfite oxidation. Biotechnol J 8: 1003-1008. 
Citation: Papagianni M (2011) Methodologies for Scale-down of Microbial Bioprocesses. J Microbial Biochem Technol S5:001. doi:10.4172/19485948.S5-001

25. Junne S, Klingner A, Kabisch J, Schweder T, Neubauer P (2011) A twocompartment bioreactor system made of commercial parts for bioprocess scaledown studies: Impact of oscillations on Bacillus subtilis fed-batch cultivations. Biotechnol J 8: 1009-1017.

26. Sweere APJ, Mesters JR, Lanse L, Luyben KCAM, Kossen NWF (1988) Experimental simulation of oxygen profiles and their influence on baker's yeast production. Part 1: one-fermentor system. Biotechnol Bioeng 31: 567-578.

27. Lin HK, Neubaueur $P(2000)$ Influence of controlled glucose oscillations on a fed-batch process of recombinant Escherichia coli. J Biotechnol 79: 27-37.

28. Hewitt CJ, Nebe-Von Caron G, Axelsson B, Mc Farlane CM, Nienow AW (2000) Studies related to scale-up of high-cell-density $\mathrm{E}$. coli fed-batch fermentations using multiparameter flow cytometry: effect of a changing microenvironment with respect to glucose and dissolved oxygen concentration. Biotechnol Bioeng 70: 381-390.

29. Oosterhuis NMG, Groesbeek NM, Olivier APC, Kossen NWF (1983) Scaledown aspects of the gluconic acid fermentation. Biotechnol Lett 5: 141-146.

30. Oosterhuis NMG, Kossen NWF, Olivier APC, Schenk ES (1985) Scale-down and optimization studies of the gluconic acid fermentation by Gluconobacter oxydans. Biotechnol Bioeng 27: 711-720.

31. Sweere APJ, Lanse L, Luyben KCAM, Kossen NWF (1988) Experimental simulation of oxygen profiles and their influence on baker's yeast production. Part 2: two-fermentor system. Biotechnol Bioeng 31: 579-586.

32. Delvigne F, Destain J, Thonard P (2006) A methodology for the design of scale-down bioreactors by the use of mixing and circulation stochastic models. Biochem Eng J 28: 256-268.

33. Dodge TC, Lundqvist PMA, Chotani GK (2011) Scale-down of production conditions in the laboratory. Chem Eng Trans 24: 901-906.

34. Baez A, Flores N, Bolivar F, Raminez OT (2011) Simulation of dissolved CO2 gradients in a scale-down system: A metabolic and transcriptional study of recombinant Escherichia coli. Biotechnol J 8: 959-967.

35. Papagianni M, Mattey M, Kristiansen B (1994) Morphology and citric acid production of Aspergillus niger PM1. Biotechnol Lett 16: 923-934.

36. Ziegler H, Dunn IJ, Bourne JR (1980) Oxygen transfer and mycelial growth in a tubular loop fermentor. Biotechnol Bioeng 22: 1613-1635.
37. Seipenbusch R, Blenke H (1980) The loop reactor for cultivating yeast on n-paraffin substrate. Adv Biochem Eng 15: 1-40.

38. Lale M (1993) Determination of time constants as a measure of bioreacto performance in a tubular loop fermenter. Ph.D. Thesis. University of Strathclyde. United Kingdom.

39. Ziegler H, Meister D, Dunn IJ, Blanch HW, Russel TWF (1977) The tubular loop fermentor: Oxygen transfer, growth kinetics and design. Biotechnol Bioeng 19 507-525.

40. Russel TWF, Bunn IJ, Blanch HW (1974) The tubular loop batch fermentor: Basic concepts. Biotechnol Bioeng 16: 1261-1272.

41. Kristiansen B, McNeil B (1987) The design of a tubular loop fermenter for scaleup and scale-down of fermentation processes. In: Moody GW, Baker PB (eds), Proc Int Conf Bioreactors and Biotransformations. Elsevier, London. p. 321.

42. Betts JI, Baganz F (2006) Miniature bioreactors: current practices and future opportunities. Microbial Cell Factories 5: 21.

43. Neubauer P, Junne S (2010) Scale-down simulators for metabolic analysis of large-scale bioprocesses. Curr Opinion Biotechnol 21: 114-121.

44. Betts JI, Doig SD, Baganz F (2006) Characterization and application of a miniature $10 \mathrm{ml}$ stirred-tank bioreactor, showing scale-down equivalence with a conventional $7 \mathrm{~L}$ reactor. Biotechnol Progr 22: 681-688.

45. Doig SD, Pickering SCR, Lye GJ, Baganz F (2005) Modelling surface aeration rates in shaken microtitre plates using dimensionless groups. Chem Eng Sci 60: $2741-2750$

46. Gill NK, Appleton M, Baganz F, Lye GL (2008) Quantification of power consumption and oxygen transfer characteristics of a stirred miniature bioreactor for predictive fermentation scale-up. Biotechnol Bioeng 100: 1144 1155.

47. Gill NK, Appleton M, Baganz F, Lye GL (2008) Design and characterization of a miniature stirred bioreactor system for parallel microbial fermentations. Biochem Eng J 39: 164-176.

48. Gill NK, Appleton M, Baganz F, Lye GL (2008) Thermal profiling for parallel online monitoring of biomass growth in miniature stirred bioreactors. Biotechnol Lett 30: 1571-1575.

This article was originally published in a special issue, Bioreactors

Modelling \& Simulation handled by Editor(s). Dr. Chongxuan Liu,

Directorate Pacific Northwest National Laboratory, USA; Dr. Nitin A.

Gawande, The Pennsylvania State University, USA 